\title{
Ni, Cu Nanoparticles Decorating CNT as Precursors for Metal-Matrix Nanocomposites
}

\author{
Mendoza, M. E. *, Solórzano, I. G. *, Brocchi, E. A. * \\ * DEMa, Department of Materials Science and Metallurgy PUC-Rio, Rio de Janeiro, P.O. 38097 Brazil
}

The increasing interest in nanostructure materials in recent years has provided incentive to develop new kind of composites containing carbon nanotubes (CNT). Such motivation relies on the wellestablished superior mechanical and transport properties of CNTs and their stability when submitted to thermo-mechanical processing together with the metal powder $[1,2,3,4,5]$.

This work reports nano-scale structural characterization of $\mathrm{Ni}$ and $\mathrm{Cu}$ decorating $\mathrm{CNT}$ synthesized by chemical method. The former was produced by dissociation of a homogeneous suspension containing $\mathrm{Ni}\left(\mathrm{NO}_{3}\right)_{2} \cdot 3 \mathrm{H}_{2} \mathrm{O}-\mathrm{MWCNT}$ and the older was produced by dissociation of a homogeneous suspension containing $\mathrm{Cu}\left(\mathrm{NO}_{3}\right)_{2} .3 \mathrm{H}_{2} \mathrm{O}-2 \mathrm{wt} \% \mathrm{SWCNT}$ with an anionic tensoactive and $\mathrm{Cu}\left(\mathrm{NO}_{3}\right)_{2} .3 \mathrm{H}_{2} \mathrm{O}-2 \mathrm{wt} \% \mathrm{MWCNT}$; followed by hydrogen reduction of the obtained NiOMWCNT and $\mathrm{CuO}-\mathrm{SWCNT}$ product.

Transmission Electron Microscopy and STEM EDS has been used as main characterization tools. The former have shown a good attachment, with measurable dihedral lower angle, of the $\mathrm{Ni}$ and $\mathrm{Cu}$ nanoparticles on to CNT, brought about by the insitu dissociation and reduction procedure of the metal nanoparticles together with the CNTs, as shown in figure 1. The later has confirmed, trough elemental mapping, the presence of $\mathrm{Ni}, \mathrm{C}$ and some residual $\mathrm{O}$, as shown in figure 2 . The Ni powder particles were observed to be in the 4-40nm range, while the $\mathrm{Cu}$ powder particles were observed to range between 50-300nm in diameter. HREM images, such as Fig. 1a, reveal that oxygen is actually coating the Nickel particle, thereby suggesting it arises from an post -oxidation stage, rather than due to an incomplete $\mathrm{NiO}$ particle reduction.

Bulk Cu-SWCNT nano-composite pellets have been obtained by cold pressing under uniaxial pressure at $60 \mathrm{MPa}$, followed by isostatic pressure under $150 \mathrm{MPa}$. Sintering of the compacted material was carry out at $650^{\circ} \mathrm{C}$ under Argon atmosphere for $15 \mathrm{~min}$. Ours observations have also shown a heterogeneous grain growth of Cu-CNT pellets with sizes between $150 \mathrm{~nm}-3 \mu \mathrm{m}$. Low temperature electric resistivity measurements, show that the nanocomposite material exhibit a lower value $\left(2 \times 10^{-6} \Omega . \mathrm{cm}\right)$ at $83 \mathrm{~K}$ as compared with copper pellets produced to the same route, however without CNT $\left(6 \times 10^{-6} \Omega . \mathrm{cm}\right)$. Hardness and elastic modulus were determined trough nanoindentation measurements. The composite displayed higher hardness $(1,7 \mathrm{GPa})$ compared with copper $(1,2 \mathrm{GPa})$ (see figure 3). Similar values were reported by J.P. Tu et al [5]. Hot compactation, variation of volume fraction and properties measurements are currently in progress.

\section{References}

[1] Brocchi, E. M.S. Motta. I.G. Solórzano. Materials Science and Engineering B 112 (2004)p200.

[2] K. T. Kim, et al. Materials science and engineering A 430 ( 2006) 27-33,.

[3] K.Tae, et al. Materials science \& Engineering A 449-451 (2007)46-50.

[4] P. Ayala, et al. Chemical Physics Letters, 431 (2006) 104-109

[5] J.P. Tu et al. Tribological letters 10, No 4, (2001) 225-228, 

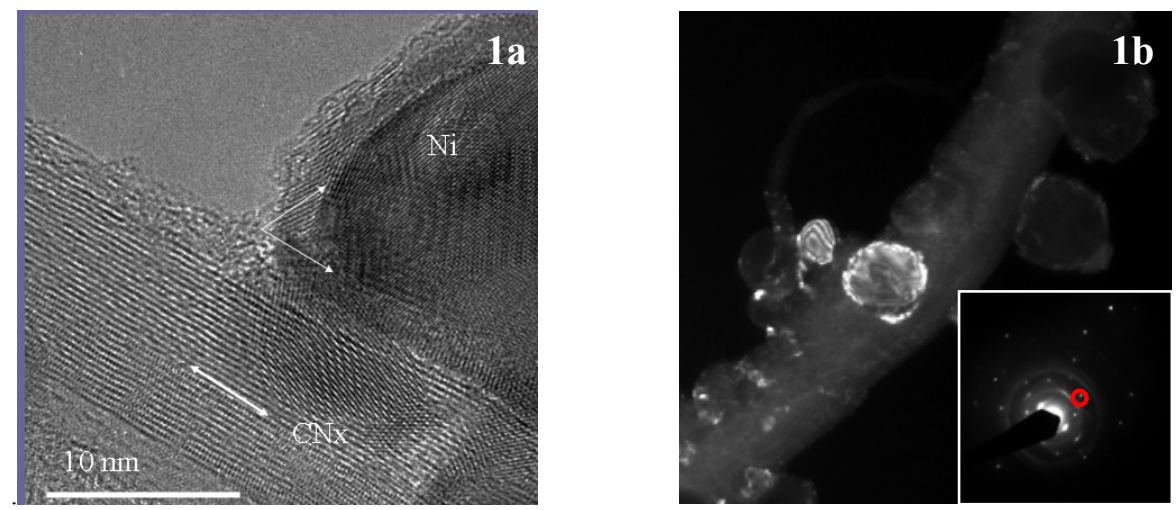

Figure 1: a) High Ressolution TEM image of Ni nanoparticle attached onto CNT b) Center dark field and diffraction pattern of Cu-MWCNT.

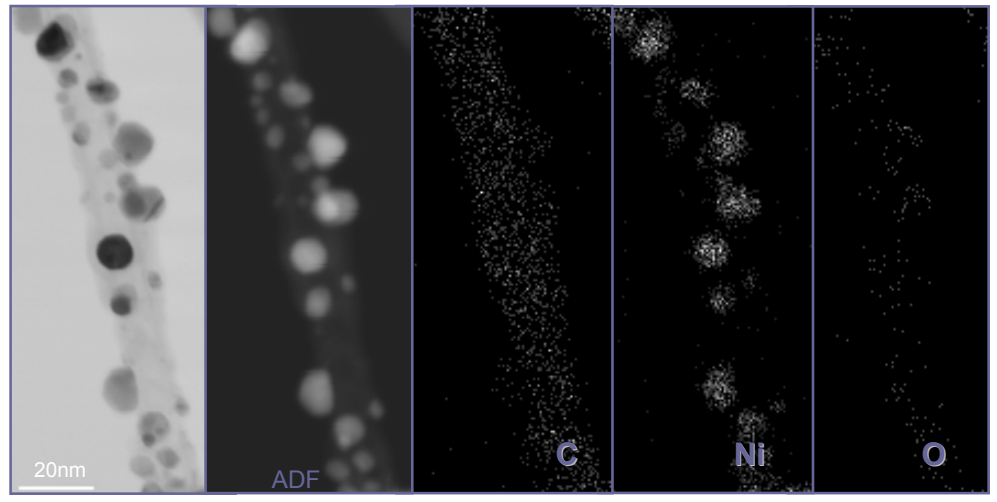

Figure 2: Bright field / Annular dark field STEM images and elemental mapping of Ni nanoparticles decorating MWCNT.
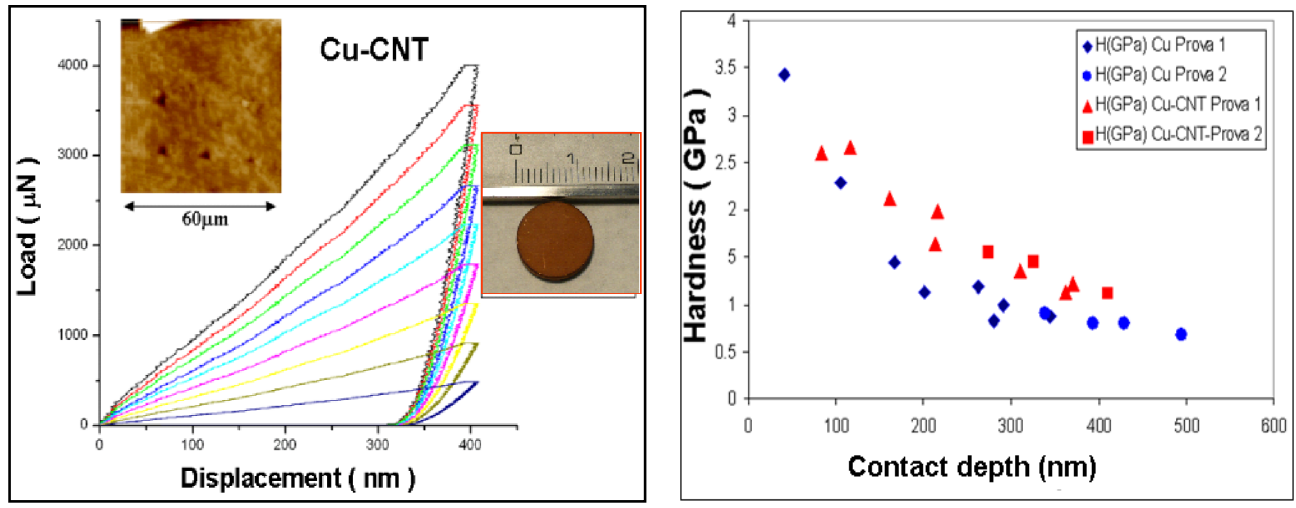

Figure 3. Hardness evaluated by Nanoindentation of a $\mathrm{Cu}-\mathrm{SWCNT}$ nanocomposite 